\title{
Microhardness of different resin cement shades inside the root canal
}

\author{
Valeria Vignolo ${ }^{1}$, Maria-Victoria Fuentes ${ }^{2}$, Miguel-Angel Garrido ${ }^{3}$, Jesús Rodríguez ${ }^{4}$, Laura Ceballos ${ }^{5}$
}

${ }^{1}$ DDS, Department of Stomatology. Rey Juan Carlos University

${ }^{2}$ DDS, PhD, Assistant Professor, Department of Stomatology. Rey Juan Carlos University

${ }^{3} \mathrm{PhD}$, Associate Professor, Department of Material Sciences and Engineering. Rey Juan Carlos University

${ }^{4} \mathrm{PhD}$, Full Professor, Department of Material Sciences and Engineering. Rey Juan Carlos University

${ }^{5}$ DDS, PhD, Associate Professor, Department of Stomatology. Rey Juan Carlos University

Correspondence:

Rey Juan Carlos University

Avda. de Atenas $s / n$

28922 Alcorcón, Madrid (Spain)

laura.ceballos@urjc.es

Received: 13/07/2011

Accepted: 27/11/2011

\author{
Vignolo V, Fuentes MV, Garrido MA, Rodríguez J, Ceballos L. Micro- \\ hardness of different resin cement shades inside the root canal. Med Oral \\ Patol Oral Cir Bucal. 2012 Sep 1;17 (5):e859-64. \\ http://www.medicinaoral.com/medoralfree01/v17i5/medoralv17i5p859.pdf \\ Article Number: $17802 \quad$ http://www.medicinaoral.com/ \\ (C) Medicina Oral S. L. C.I.F. B 96689336 - pISSN 1698-4447 - eISSN: 1698-6946 \\ eMail: medicina@medicinaoral.com \\ Indexed in: \\ Science Citation Index Expanded \\ Journal Citation Reports \\ Index Medicus, MEDLINE, PubMed \\ Scopus, Embase and Emcare \\ Indice Médico Español
}

\begin{abstract}
Objectives: To compare microhardness along the root canal post space of two resin cements in different shades and a dual-cure resin core material.

Study Design: Root canals of 21 bovine incisors were prepared for post space. Translucent posts (X・Post, Dentsply DeTrey) were luted using one the following resin luting agent: Calibra (Dentsply DeTrey) in Translucent, Medium and Opaque shades, RelyX Unicem (3M ESPE) in Translucent, A2 and A3 shades and the dual-cure resin core material Core $X$ flow. All materials were applied according to manufacturers' instructions and were all photopolymerized (Bluephase LED unit, Ivoclar Vivadent, 40s). After 24 hours, roots were transversally cut into 9 slices 1 $\mathrm{mm}$ thick from the coronal to apical extremes, three corresponding to each root third. Then, VHNs were recorded (100gf, $30 \mathrm{~s})$ on the resin luting materials along the adhesive interface in all sections. Data were analyzed by twoway ANOVA and SNK tests $(\alpha=0.05)$.

Results: A significant influence on microhardness of resin luting material in their respective shades $(\mathrm{p}<0.001)$, root third $(p<0.001)$ and interactions between them was detected $(p<0.001)$. RelyX Unicem cement showed the highest microhardness values and Calibra the lowest, regardless of the shade selected. All resin luting materials tested exhibited a significantly higher microhardness in the cervical third.

Conclusions: Microhardness of resin luting agents tested inside the canal is dependent on material brand and resin cement shade seems to be a less relevant factor. Microhardness decreased along the root canal, regardless of the shade selected.
\end{abstract}

Key words: Cement shade, degree of conversion, dual-cured resin cements, fiber posts, microhardness, root thirds. 


\section{Introduction}

Nowadays, translucent fiber reinforced composite posts constitute the first option to restore non-vital teeth with an excessive loss of coronal structure (1). Besides good esthetics, their main advantage is that their elastic modulus is similar to dentin (2), inducing a favorable distribution of stress which prevents root fractures (1). In order to achieve this homogeneous biomechanical unit, the use of resin cements is utterly necessary to lute the posts into the roots. Clinicians can choose among resin cements that require the previous application of an adhesive system, self-adhesive resin cements or even dual-cure resin core materials (3).

In all cases, the luting agent selected must be a dualcure resin material in order to compensate light attenuation, as most of the posts are cemented to an extent of 8 to $10 \mathrm{~mm}(4,5)$. However, the polymerization of some dual-cure resin cements has been reported to be mainly dependent on light exposure (6), whilst the chemicalcuring method, when acting alone, is considered to be slower, less effective and not capable of compensating the attenuation of light $(6,7)$.

Therefore, the use of translucent fiber posts has been also recommended as they transmit light to deeper depths allowing the activation of light-polymerizing components of the dual-cure resin cements $(4,6,8)$ and, thus, improving their degree of conversion (6).

The polymerization efficacy of resin materials has been also related to characteristics of the material itself, such as chemical composition, filler particle size, and shade and translucence $(9,10,11)$. The darker shades have been reported to exhibit a reduction in light transmission not curing as deeply as lighter shades $(12,13)$. Besides, more translucent materials are considered to allow better light transmission which would result in a higher degree of conversion. Although this circumstance has been described for restorative resin composites (14), no references have been found regarding this possible effect on resin cements polymerization efficacy when used to lute fiber posts.

An adequate degree of conversion of the resin cement is mandatory to ensure that physical and mechanical properties are good enough to withstand masticatory forces immediately after cementation while maintaining an adequate bonding and dentin sealing (15).

The degree of conversion of resin cements can be assessed by indirect methods such as microhardness testing. This being a widely accepted method as it presents a good correlation with the spectroscopy approach $(7,16)$. Therefore, the aim of this study was to compare the microhardness along the root canal post space of: a resin cement that requires the previous application of an adhesive system; a self-adhesive resin cement; and a dual-cure resin core material. The later is available in only one shade while the formers were tested in 3 different shades. The null hypotheses tested were: the three resin luting materials exhibit similar microhardness values regardless of the shade; and microhardness is uniform along the root canal depth.

\section{Material and Methods}

Twenty one bovine incisors teeth were sectioned at 16 $\mathrm{mm}$ from the root apex to standardize the root length. The pulp was then removed with \#90-K files (Maillefer, Ballaigues, Switzerland).

A $12 \mathrm{~mm}$-deep post space was prepared in each root with the drills provided by the manufacturer of the posts (X·Post, Size 3, Dentsply DeTrey, Konstanz, Germany), leaving $4 \mathrm{~mm}$ in the apical portion. In all cases, the translucent fiber posts X•Post (Size 3: length $20 \mathrm{~mm}$, tip diameter $0.8 \mathrm{~mm}$ and head diameter $1.67 \mathrm{~mm}$, Dentsply DeTrey) were used. They were all cut at $16 \mathrm{~mm}$ and cleaned with alcohol after try-in.

Before cementation procedures, the external lateral walls of the roots were painted with two layers of black nail varnish to avoid any external light curing. Afterwards, post spaces were rinsed with $2.5 \%$ sodium hypochlorite irrigation and dried with absorbent paper points.

The roots were randomly allocated to seven groups according to the resin luting agent used and its shade. The dual-cure resin materials evaluated were: Calibra in Translucent, Medium and Opaque shades (Dentsply DeTrey), which is a resin cement that uses an etch and rinse adhesive system (Prime \& Bond NT, Dentsply DeTrey); RelyX Unicem in Translucent, A2 and A3 shades (3M ESPE, Seefeld, Germany), which is a self-adhesive resin cement and Core $\times \mathrm{X}$ flow (Dentsply DeTrey) which is a composite core material that can be also used as luting material and available only in one shade and uses an etch and rinse adhesive system (XP Bond, Dentsply DeTrey). Chemical composition of the adhesive systems and resin luting materials tested are shown in (Table 1). They were all used following manufacturers' instructions.

The resin luting materials were all photopolymerized for $40 \mathrm{~s}$ with a LED unit (Bluephase, Ivoclar Vivadent, Schaan, Liechtenstein) applying the high intensity program $\left(1200 \mathrm{~mW} / \mathrm{cm}^{2}\right)$. The tip of the light unit was kept directly in contact with the coronal end of the post.

Afterwards, specimens were included in resin blocks (Epoxicure Resin-Hardener, Buehler, Lake Bluff, IL, USA) and stored for $24 \mathrm{~h}$ in light-proof containers, at $37^{\circ} \mathrm{C}$ until testing. Roots were then sectioned perpendicular to their long axis with a diamond blade (Accutom-5, Struers, Copenhagen, Denmark), under irrigation. Nine serial slices $1 \mathrm{~mm}$ thick were obtained from the coronal to apical extremes, three corresponding to each root third. Surfaces to be analyzed were polished with 4000-grit $\mathrm{SiC}$ papers for $30 \mathrm{~s}$ at $300 \mathrm{rpm}$ (Labopol-5, Struers). 
Table 1. Chemical composition of the resinous cements and adhesive systems.

\begin{tabular}{|c|c|}
\hline Cement & Adhesive system \\
\hline $\begin{array}{l}\text { RelyX Unicem ( } 3 \mathrm{M} \text { ESPE) } \\
\text { Powder: glass particles, iniciators, silica, substituted } \\
\text { pyrimidine, calcium hydroxide, peroxide composite and } \\
\text { pigments. } \\
\text { Liquid: methacrylate phosphoric acid ester, dimethacrylate } \\
\text { acetate, stabilizer and iniciator. }\end{array}$ & Does not require \\
\hline $\begin{array}{l}\text { Calibra (Dentsply DeTrey) } \\
\text { Calibra Esthetic Resin Cement Base: Dimethacrylate } \\
\text { Resins;Camphorquinone (CQ) Photoinitiator; Stabilizers; } \\
\text { Glass Fillers; Fumed silica; Titanium Dioxide; Pigments } \\
\text { Calibra Esthetic Resin Cement Catalyst: Dimethacrylate } \\
\text { Resins; Catalyst; Stabilizers; Glass Fillers; Fumed Silica }\end{array}$ & $\begin{array}{l}\text { Prime\&Bond NT (Dentsply DeTrey) } \\
\text { (Total-Etch technique) } \\
\text { PENTA, UDMA resin, Resin R5-62-1, T-Resin, } \\
\text { D-resin, Nanofiller, Intiators, Stabiliser, } \\
\text { Cetylamine and Hydrofluoride Acetone } \\
\text { Self-Cure Activator } \\
\text { UDMA, HEMA, Catalyst, Photoinitiators, } \\
\text { Stabilizers, Acetona, Water }\end{array}$ \\
\hline $\begin{array}{l}\text { Core } \text { X flow (Dentsply DeTrey) } \\
\text { Consists of two components, base and catalyst, is available } \\
\text { in a dual-barreled syringe. } \\
\text { Urethane Dimethacrylate, Di-\& Tri-funtional } \\
\text { Methacrylates, Barium Boron Fluoroaluminosilicate Glass, } \\
\text { Camphorquinone (CQ) Photoinitiator, Photoaccelerators, } \\
\text { Silicon Dioxide and Benzoyl Peroxide. }\end{array}$ & $\begin{array}{l}\text { XP Bond (Dentsply DeTrey) } \\
\text { (Total-Etch technique) } \\
\text { PENTA, TCB resin, UDMA, TEGDMA, HEMA, } \\
\text { Nanofiller, Camphorquinone, DMABE, Butylated } \\
\text { benzenediol Stabilises and tert-Butanol } \\
\text { Self-Cure Activator: } \\
\text { UDMA, HEMA, Catalyst, Photoinitiators, } \\
\text { Stabilisers, Acetona, Water }\end{array}$ \\
\hline
\end{tabular}

Indentations were performed on the resin luting materials along the adhesive interface between root dentin and the fiber post in all the sections obtained by means of a Vickers digital microdurometer (Buehler 2101, Buehler), applying $100 \mathrm{gf}$ during $30 \mathrm{~s}$. A minimum distance corresponding to the length of two indentations was maintained between indentations and between indentations and the post or the dentin. Hardness was expressed as Vickers hardness number (VHN).

The influence of resin luting agent used considering its shade and the root third was analyzed by two-way ANOVA. Posterior comparisons were performed by Student-Newman-Keuls test. All statistical testing was performed at a pre-set alpha of 0.05 by means of IBM SPSS 18 (IBM Company, Chicago, IL, USA).

\section{Results}

Fig. 1 shows the mean VHN obtained for each resin luting material and shade evaluated at cervical, middle and apical root third. It must be pointed out that during specimen cutting no displacement of the cemented posts was observed.

According to the results, a significant influence of resin luting material in their respective shades $(\mathrm{p}<0.001)$, root third $(\mathrm{p}<0.001)$ and interactions between them was detected $(\mathrm{p}<0.001)$.
In the three root thirds, the highest microhardness values were attained with RelyX Unicem resin cement and the lowest with Calibra, always and regardless of the shade used. Specimens luted with Calibra showed similar microhardness values for Translucent, Medium and Opaque shades in the three thirds. In contrast, several statistical differences were detected among different shades of RelyX Unicem resin cement. In the cervical third, RelyX Unicem Translucent specimens exhibited microhardness numbers significantly higher than those obtained with A2 and A3 ones. In the middle third, specimens luted with RelyX Unicem A3 showed statistically higher microhardness values than those luted with RelyX Unicem A2, but similar in both cases to the ones determined for Translucent shade. In the apical third, no statistical differences were detected among RelyX Unicem shades. Core $X$ flow microhardness results were intermediate to the ones attained with the other materials.

Regarding the variation of microhardness values of each luting material evaluated according to the root third, all of them exhibited a significantly higher microhardness in the cervical third in comparison with the other two thirds. In the apical third microhardness values significantly decreased in comparison to the middle third only with RelyX Unicem Translucent and A3 and Calibra Translucent and Medium shades. 


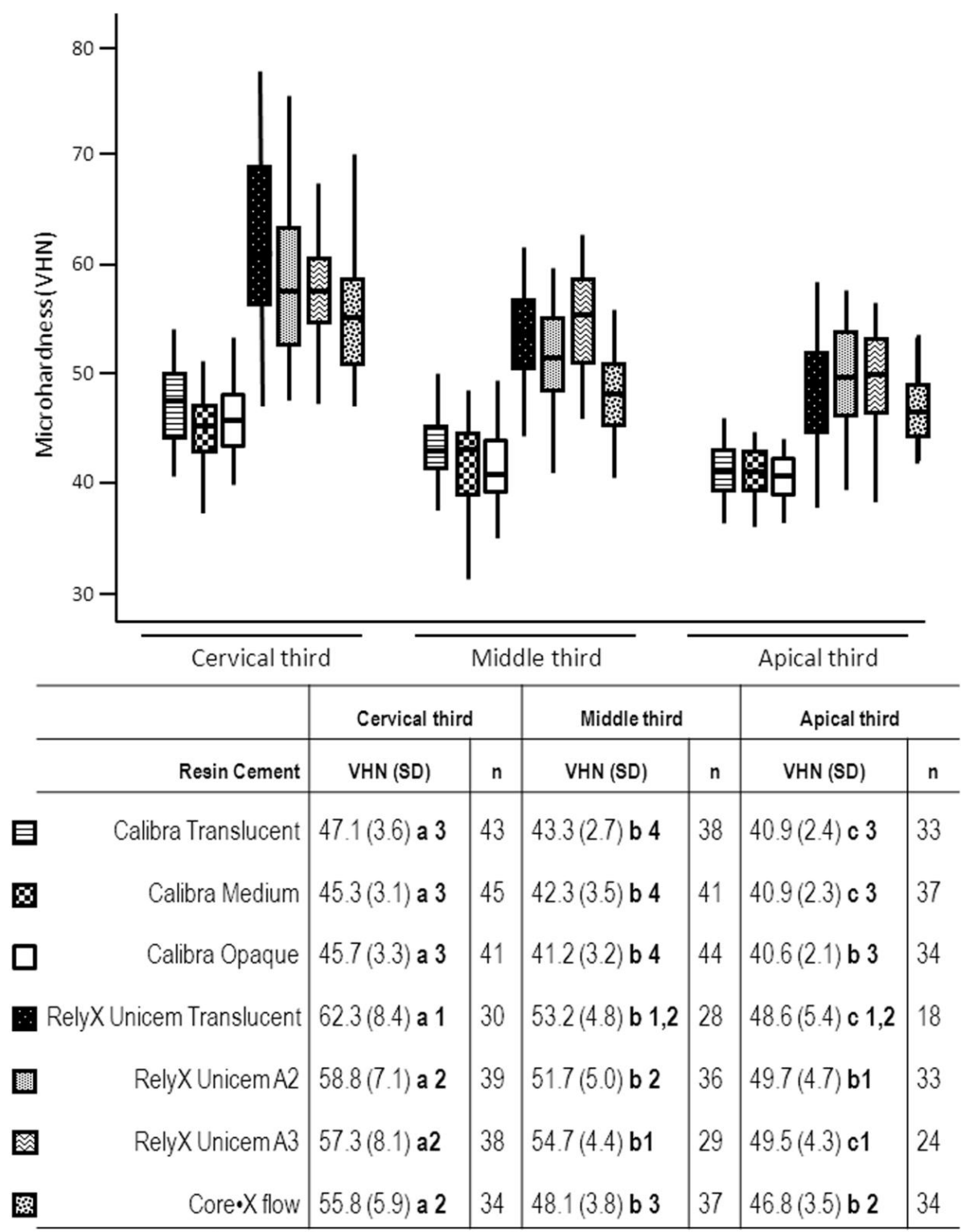

Same numbers in the same column mean no differences among resin cements at each root portion. Same letters in the same row mean similar microhardness in differentroot thirds.

Fig. 1. Means VHN and standard deviations determined for the resin luted materials tested along the root canal post space at the cervical, middle and apical thirds.

\section{Discussion}

The results of the present work indicate that the three resin luting materials tested exhibited significantly different microhardness values. Thus, microhardness is dependent on resin luting material brand. RelyX Unicem cement showed the highest microhardness values and Calibra the lowest, regardless of the shade selected. Moreover, microhardness of all luting materials evaluated significantly decreased along the root canal. Hence, the null hypotheses formulated must be rejected.

An adequate polymerization of dual-cure post luting cements is desirable to provide mechanical proper- ties good enough to clinically ensure retention of the post (17). Otherwise, all the loads would concentrate on the luting material, the component with the lowest mechanical strength in comparison with dentin and the post (18).

According to literature, microhardness is a simple testing method that can be correlated to the extent of polymerization $(15,19,20)$. However, care should be taken when comparing different resin materials' microhardness as other factors as the nature of the matrix, type of filler, filler load, the quantity of initiators, the amount of inhibitors and the ratio between auto- and light-polyme- 
rizing components strongly influence the final amount of reacted monomers $(7,20)$.

Self-adhesive resin cements constitute an attractive alternative for post cementation as no dentin pretreatment is required simplifying the procedure and reducing technique and operator sensitivity (20). Specifically, RelyX Unicem has been reported to produce an effective adhesion with dentin despite its very superficial interaction with this tissue, exhibiting similar (21) or even higher bond strength values to root dentin than conventional resin cements (3) and a better sealing ability (22). In the present study, this resin cement attained the highest microhardness values in comparison with the other two luting materials. Other authors $(20,23)$ have reported a mean microhardness value somewhat lower for this resin cement $(\mathrm{VHN}=44)$. Unlike this study, in both cited works, the luting procedure inside the root canal was not simulated. Although the dominant setting reaction starts with free radical polymerization, phosphoric acidic methacrylates, included in this resin cement, also react with either the basic fillers or the hydroxyapatite of dentin, which may contribute to a higher hardness (24). Regarding the correlation between microhardness and the degree of cure for this particular cement, information is controversial. While Cadenaro et al. (20) reported a significant correlation between both parameters, in the study by Kumbuloglu et al. (23) the highest microhardness and compressive strength were obtained by this self-adhesive resin cement in comparison with other resin-based luting cements such as Panavia F (Kuraray), Variolink 2 (Ivoclar Vivadent) and RelyX ARC (3M ESPE), but the degree of conversion was, in contrast, the lowest.

The lowest microhardness values were obtained by Calibra resin cement. Previous studies have determined a degree of cure ranging between 59 and $61.5 \%$ (25). The lower filler load of this resin cement in comparison with RelyX Unicem may explain the differences in microhardness detected between both materials. Microhardness is related to other mechanical properties as flexural strength (26) and according to our results, in an in vitro study (27), showed that RelyX Unicem obtained higher flexural strength than Calibra after 1-day storage.

In the present study, Core $X$ flow exhibited intermediate values statistically similar in the cervical third to the ones obtained by RelyX Unicem A2 and A3, and to RelyX Unicem Translucent in the apical third. In the middle third they were intermediate and statistically different to the values determined for Calibra and RelyX Unicem resin cements. It should be mentioned that there is no literature regarding its microhardness, degree of cure, bonding effectiveness or sealing ability.

According to our results, changes in microhardness among different shades of the same resin cements have been only significant in cervical and middle root thirds for RelyX Unicem resin cement. In the apical root third, where light intensity inside the canal seems to decrease to levels insufficient for light polymerization, no differences among resin cement shades were detected. Therefore, its influence varies with resin cement brand and root third. In any case, resin cement brand and root third seem to be more relevant than the shade selected for the microhardness values obtained.

Lighter shades are considered to cure more deeply than darker ones due to an increased capacity for light to penetrate into the bulk of the composite, since pigments in darker shades might absorb more light and reduce its penetration into the resin (12). Nevertheless, translucence seems to be a much more influential predictor of cure depth than any color or shade change in material on curing (9).

In contrast with literature regarding the relevance of shade of resin composites lighter and more translucent shades were not related to significantly higher microhardness values $(13,28)$. In the present study, only RelyX Unicem Translucent in the cervical third showed higher microhardness than the same cement in A2 and A3 shades and no differences were detected among Calibra shades in any root third.

It must be taken into account that only two resin cements in different shades and only one fiber post brand and one light polymerization procedure were tested. Therefore, other results might be obtained with other resin luting materials. In any case, comparison between different resin luting materials would not be easy as usually shades are keyed to the Vita Classic Shade guide, but some brands distinguish shades according to value, hue or translucency. Moreover, large quantitative color differences have been detected among resin composites of the same Vita shade (27).

Regarding the variation of microhardness values of each luting material tested according to the root third, all of them exhibited statistically higher microhardness values in the cervical third. Previous works have determined that the tendency of degree of conversion to decrease within the root canal depends on the type of post $(6,29,30,31)$, considering translucent posts the best option to enhance light transmission $(4,6)$. No reference has been found regarding the ability of the posts tested to transmit light. However, Alves et al., (29) evaluated ten fiber posts of different manufacturers and translucencies, and for all posts a significant reduction in the quantity of light transmitted as the depth increased was revealed. According to these authors and Galhano et al., (30) the light transmission of the translucent posts in the deeper regions would be insufficient for clinical luminous activation of resin. Therefore, the three resin luting materials tested would still be dependent on light activation, with the proximity of the light curing unit playing a key role in their degree of conversion. The 
decrease in microhardness values from coronal to apical has been previously described for Calibra resin cement (31). It has been reported by Arrais et al., (40) that the fifth generation dual cure luting systems, as Calibra, contain a lower quantity of self-curing components that may not be capable of compensating for the decrease in light intensity.

On the other hand, Pedreira et al., (24) reported a homogeneous microhardness for RelyX Unicem along the root canal. However, methodological differences may have influenced as, in our study, the measurements were performed after $24 \mathrm{~h}$ and in their study after 7 days water storage, allowing a more intense influence of the root canal environment on RelyX Unicem curing.

The authors are aware this study focus in one type of fiber post, one light-curing procedure and only three resin luting materials. Therefore, the results obtained should not be extrapolated to other materials. Even so, according to our measurements, resin cement shade seems to be a less relevant factor on microhardness. Finally, this study aims to determine microhardness as an indirect measurement of curing degree and no value has been establish to ensure clinical success. Therefore, high microhardness values are not necessarily related with a good clinical performance.

\section{References}

1. Naumann M, Blankenstein F, Dietrich T. Survival of glass fibre reinforced composite post restorations after 2 years-an observational clinical study. J Dent. 2005;33:305-12.

2. Plotino G, Grande NM, Bedini R, Pameijer CH, Somma F. Flexural properties of endodontic posts and human root dentin. Dent Mater. 2007;23:1129-35.

3. Bitter K, Paris S, Pfuertner C, Neumann K, Kielbassa AM. Morphological and bond strength evaluation of different resin cements to root dentin. Eur J Oral Sci. 2009;117:326-33.

4. Yoldas O, Alacam T. Microhardness of composites in simulated root canals cured with light transmitting posts and glass-fiber reinforced composite posts. J Endod. 2005;31:104-6.

5. Ceballos L, Garrido MA, Fuentes V, Rodriguez J. Mechanical characterization of resin cements used for luting fiber posts by nanoindentation. Dent Mater. 2007;23:100-5.

6. Kim YK, Kim SK, Kim KH, Kwon TY. Degree of conversion of dual-cured resin cement light-cured through three fiber posts within human root canals: an ex vivo study. Int Endod J. 2009;42:667-74.

7. Hofmann N, Papsthart G, Hugo B, Klaiber B. Comparison of photo-activation versus chemical or dual-curing of resin-based luting cements regarding flexural strength, modulus and surface hardness. J Oral Rehabil. 2001;28:1022-8.

8. Roberts HW, Leonard DL, Vandewalle KS, Cohen ME, Charlton DG. The effect of a translucent post on resin composite depth of cure Dent Mater. 2004;20:617-22.

9. Shortall AC. How light source and product shade influence cure depth for a contemporary composite. J Oral Rehabil. 2005;32:90611.

10. Musanje L, Darvell BW. Curing-light attenuation in filled-resin restorative materials. Dent Mater. 2006;22:804-17.

11. Reges RV, Costa AR, Correr AB, Piva E, Puppin-Rontani RM, Sinhoreti MA, et al. Effect of light-curing units, post-cured time and shade of resin cement on knoop hardness. Braz Dent J. 2009;20:410-3
12. Leloup G, Holvoet PE, Bebelman S, Devaux J. Raman scattering determination of the depth of cure of light-activated composites: influence of different clinically relevant parameters. J Oral Rehabil. 2002;29:510-5.

13. Briso AL, Fedel TM, Pereira S de M, Mauro SJ, Sundfeld RH, Sundefeld ML. Influence of light curing source on microhardness of composite resins of different shades. J Appl Oral Sci. 2006;14:10-5. 14. Reges RV, Moraes RR, Correr AB, Sinhoreti MA, Correr-Sobrinho L, Piva E, et al. In-depth polymerization of dual-cured resin cement assessed by hardness. J Biomater Appl. 2008;23:85-96.

15. Attar N, Tam LE, McComb D. Mechanical and physical properties of contemporary dental luting agents. J Prosthet Dent. 2003;89:127-34.

16. Rueggeberg FA, Craig RG. Correlation of parameters used to estimate monomer conversion in a light-cured composite. J Dent Res. 1988;67:932-7.

17. Giachetti L, Scaminaci Russo D, Bertini F, Giuliani V. Translucent fiber post cementation using a light-curing adhesive/composite system: SEM analysis and pull-out test. J Dent. 2004;32:629-34.

18. Al-Assaf K, Chakmakchi M, Palaghias G, Karanika-Kouma A, Eliades G. Interfacial characteristics of adhesive luting resins and composites with dentine. Dent Mater.2007;23:829-39.

19. Pereira SG, Osorio R, Toledano M, Nunes TG. Evaluation of two Bis-GMA analogues as potential monomer diluents to improve the mechanical properties of light-cured composite resins. Dent Mater. 2005;218:823-30

20. Cadenaro M, Navarra CO, Antoniolli F, Mazzoni A, Di Lenarda $\mathrm{R}$, Rueggeberg FA, et al. The effect of curing mode on extent of polymerization and microhardness of dual-cured, self-adhesive resin cements. Am J Dent. 2010,23:14-8.

21. Zicari F, Couthino E, De Munck J, Poitevin A, Scotti R, Naert I, et al. Bonding effectiveness and sealing ability of fiber-post bonding. Dent Mater. 2008;24:967-77.

22. Santos V, Perdigao J, Gomes G, Silva AL. Sealing ability of three fiber dowel systems. J Prosthodont. 2009;18:566-76.

23. Kumbuloglu O, Lassila LV, User A, Vallittu PK. A study of the physical and chemical properties of four resin composite luting cements. Int J Prosthodont. 2004;17:357-63.

24. Pedreira AP, Pegoraro LF, de Goes MF, Pegoraro TA, Carvalho RM. Microhardness of resin cements in the intraradicular environment: effects of water storage and softening treatment. Dent Mater. 2009;25:868-76.

25. Arrais CA, Rueggeberg FA, Waller JL, de Goes MF, Giannini M. Effect of curing mode on the polymerization characteristics of dualcured resin cement systems. J Dent. 2008;36:418-26.

26. Borba M, Della Bona A, Cecchetti D. Flexural strength and hardness of direct and indirect composites. Braz Oral Res. 2009:23:5-10. 27. Irie M, Maruo Y, Nishigawa G, Suzuki K, Watts DC. Physical properties of dual-cured luting-agents correlated to early no interfacial-gap incidence with composite inlay restorations. Dent Mater. 2010;26:608-15.

28. Anfe TE, Caneppele TM, Agra CM, Vieira GF. Microhardness assessment of different commercial brands of resin composites with different degrees of translucence. Braz Oral Res. 2008;22:358-63.

29. dos Santos Alves Morgan LF, Peixoto RT, de Castro Albuquerque R, Santos Correa MF, de Abreu Poletto LT, Pinotti MB. Light transmission through a translucent fiber post. J Endod. 2008;34:299-302. 30. Galhano GA, de Melo RM, Barbosa SH, Zamboni SC, Bottino MA, Scotti R. Evaluation of light transmission through translucent and opaque posts. Oper Dent. 2008;33:321-4.

31. Radovic I, Corciolani G, Magni E, Krstanovic G, Pavlovic V, Vulicevic ZR, et al. Light transmission through fiber post: the effect on adhesion, elastic modulus and hardness of dual-cure resin cement. Dent Mater. 2009;25:837-44.

\section{Acknowledgments}

The authors thank the manufacturers for supplying materials for this study. This research was supported in part by URJC-CM-2008-BIO3526 . 